\title{
Halsmarkschädigung und Versehrung der A. vertebralis.
}

\author{
Von Prof. J. Wieting, Sahlenburg.
}

Es wäre unphysiologisch gedacht, für jede funktionelle Störung, selbst den Tod, in jedem Falle eine grob oder fein erkennbare anatomische Grundlage finden $\mathrm{zu}$ wollen. Das darf aber nicht hindern, in jedem Falle nach einer solchen $z u$ suchen, und es gibt ein Gefühl der Befrjedigung, wenn wir sie finden, und je feiner unsere Methodik der pathologisch-anatomischen Untersuchungen und je exakter unsere klinische Beobach. tung, desto öfter werden wir sie finden.

Für die örtliche Veränderung durch das Gehirn nicht unmittelbar treffende Gewalten nehmen wir als Ursache eine Kontusion an, sei es durch momentane Formveränderung der elastischen Hirnschale, sei es durch molekulare Erschütterung oder Fernwirkung. Diese ist bei den konstruktiven Verhältnissen der Hirnschale zu ihrem Inhalt wohl verständlich; die Einwirkung ist unmittelbar und demnach auch das klinische Geschehen. Progressive Veränderungen sind als Komplikationen aufzufassen. Der anatomische Ausdruck ist der primäre örtliche, nicht selten auch der fernörtliche Erw e i c hu n g s h e rd, mit stets deutlich erkennbarer hämorrhagischer Beimischung.

Ähnliches hat man nun auch für das $\mathrm{Hals}$ - und $\mathrm{R}$ ü ckenmark angenommen. Bei der sogenannten Erschütterung ist meistens eine anatomische Veränderung nicht nachweisbar. H. Heinecke (s. „Verletzungen der Wirbelsäule“ in Borchard-Schmiedens Kriegschirurgie 1917) spricht von Erschütterungswirkungen, als ,vielleicht rein zirkulatorischen Störungen, Ödemen, die wohl imstande sein können, die Funktion vorübergehend lahm zu legen, ohne dieselbe dauernd zu schädigen". Indessen werden, auch ohne daß das Rückenmark selber unmittelbar getroffen wurde, z.B. nach Schußgewalt-Seitendruck- 
wirkung (Prellschüsse), etwa auf die Dornfortsätze oder Wirbelbögen nicht selten mehr oder minder $\mathrm{sch}$ were Erweich un gen der Rückenmarksubstanz beschrieben (s. Borst in Borchard-Schmiedens Kriegschirurgie und Reese ${ }^{1}$ ), die als molekulare Erschütterungswirkung angesehen werden. Von einer Druckwirkung infolge Formveränderung einer elastisch knöchernen Kapsel, etwa wie am Gehirn, kann hier nicht wohl die Rede sein; wir müssen hier eben eine Erschütterungswelle, die sich von dem Knochen auf die den Wirbelkanal ausfüllenden flüssigen und wcichen Bestandteile fortpflanzt, annehmen, werden aber als Ausdruck dieser Erschütterung einmal anatomisch auch der Wirkung auf die feinen Gefäße, die dem Erweichungsherd hämorrhagisches Aussehen gibt, nicht vergessen und zum anderen klinisch die sofortige funktionelle Störung erwarten dürfen. Die Annahme, daß nach Gewalteinwirkungen Kontusion zunächst die feinen Gefäße in ihren Zellen fettig oder gar atherosklerotisch entarten, und danach erst die Erweichung

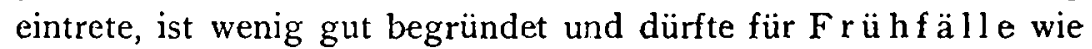
den folgenden kaum in Erwägung gezogen werden können.

Eine andere Erklärung erfordern die Fälle, in denen die Erweichung anämisches, nicht hämorrhagis ches A ussehen - auch nicht in feinster Schattierung - zeigt und in dem die klinischen Erscheinungen nicht un. mittelbarim Anschluß an die Verletzung, sondern nach kürzerem oder längerem Intervall ziemlich plötzlich einsetzen und durch ihre Schwere verhängnisvoll werden können. Eine solche Beobachtung sei hier kurz mitgeteilt:

Der 19 j. Infanterist K. J. erlitt am II. XI. 1917 nachmittags gegen 5 Uhr eine Granatsplittcrverletzung mit EinschuB in di er. Wange vor dem Ohre. Am 12. XI. B efund: Vor dem $r$. Ohr eine fingergliedgroße dreieckige Weichteilwunde; die r. Gesichtshälfte stark geschwollen, ebenso die $r$. Halsseite mit braunblauer Hautfärbung. Im Munde ist die r. Mandelgegend stark geschwollen und gerötet, doch gestattet die Kieferklemme keine Übersicht, ob hier ein AusschuB vorhanden ist. Das Bewu Bts e in ist ungestört, die Sprache heiser, was sie aber, nach Angabe des

I) H. Reese, Uber Geschoß-Seitendruckwirkungen auf das Rückenmark in der Diagnose und Behandlung der Spätfolgen von Kriegsverletzungen. Zürich 1920. Deut. Zeitschr. f. Chir. Bd. 158. 1920. 
Verletzten, schon immer gewesen sei. Außer rechtsseitiger peripherer Fascialislähmung keine bemerkenswerten nervösen Stör ung en, auch nicht solche peripherer Art. Die Wangenwunde führt auf zerschmetterten Unterkieferknochen.

Abends Überführung nach der Kriegslazarett-Kieferabteilung. Am 13. XI. Heute früh starke Blutung a us der Wunde, die zunächst auf Jodoformmullstopfung steht. Zunächst Verdacht auf Carotis externa-Verletzung, besonders da das obere Halsdreieck stark, wenn auch ohne Pulsation geschwollen ist. Das $\mathrm{R}$ öntge nbild zeigt einen $\mathrm{I}: \mathrm{I} 1 / 2 \mathrm{~cm}$ großen Granatsplitter in Projektion von vorn nach hinten etwas r. von der Mittellinie, vor dem 5. Halswirbel.

Operation (Wieting): Fingeraustastung des Kanals führt gegen die großen Gefäße, daher Freilegung der Carotis communis durch Sonderschrägschnitt vor dem r. Kopfnicker. Die ganze Umgebung, auch die Gefäßscheide, ist blutig durchtränkt. Die Teilungsstelle wird sichtbar gemacht, ohne daß eine Blutung aus der Operationswunde eintritt. Nach Entfernung der Mullstopfen aus der Schußwunde tritt hier starke Blutung ein, daher zunächst Unterbindung der Carotis externa, deren Sperrung auf alle Fälle bei der gleichzeitigen Kieferverletzung geboten erschien. Die Blutung steht nicht, daher wird nach digitaler Kompression der Carotis communis, die Schnittwunde nach oben erweitert und die Carotis communis wegen der Gefahr der Ausblutung zunächst temporär abgebunden. $\mathrm{Da}$ es trotzdem weiter blutet, wird die allein noch bestehende und offenbar aus peripherer Richtung blutende Carotis interna abgeklemmt, die Klemmen liegen gelassen. Die Unterbindung der Carotis communis bleibt liegen, da sie jetzt belanglos ist, aber die Klemmen sichert. Der Granatsplitter wird mit der Kornzange entfernt und dabei getastet, da $B$ ein (der 4. oder 5. ?) Halswirbel r. seitlich verletzt ist. Die Blutung steht unter Mullstopfung, aber nach der Sachlage besteht der Verdacht, daß außer der Carotis interna auch die rechte Vertebralis verletzt sei.

Danach erwacht der Pat., wird aber in der Nacht zum I4. XI. sehr unruhig und hat schwere Atemnot, wie man sie nach Verletzung des oberen Halsmarkquerschnittes beobachtet. Am Morgen tritt der Tod ein.

Die Sektion ergibt die obigen Gefäßunterbindungen. Dic Vena jugularis ist unverletzt, die Carotis interna ist auf $\mathrm{I}^{1 / 2} \mathrm{~cm}$ zerrissen, mit einer schmalen Brücke zwischen den fetzigen Rändern, die beiderseits noch die Abklemmungsmarken tragen. Im oberen Teil sitzt ein kleiner lockerer Plättchenpfropf, der N. va g u s ist gestreift mit seitlicher Auffaserung. Die $\mathrm{C}$ a r o t is externa und die communis sind nicht verletzt. Der Seitenfortsatz des 4 . Halswirbels ist rechts grubig eingedrückt, da, wo der Granatsplitter $\mathrm{saB}$. 
DieA. vertebralis dextra ist an der Vorderwand fet $z$ ig zerrissen, ein Bruch des Wirbelbogens nicht sicher festzustellen. Im Wirbelkanal keine Blutung, keine Verletzung der Dura, auch im Durasack kein Blut. En tsprechend dem 4. Hals. wirbel ist das $\mathrm{Halsmark}$ weich und drängt spindelförmig vor. Eine Blutung auch hier nicht festzustellen. Auf dem Querschnitt ist oberhalb und unterhalb der hervorquellenden Erweichungsstelle Zeichnung und Beschaffenheit normal.

AuBer starkem Lunge nö dom ergibt die Sektion keinen besonderen Befund. (Leider ist die A. vertebralis in ihrem weiteren Verlauf nicht genau untersucht.) Anatomische T odesursache: Bruch des Seitenfortsatzes des 4. Halswirbels mit Verletzung der $r$. A. vertebralis und Kontusionserweichung im Halsmark.

Die Halsmarkerweichung in diesem Falle auf Fernkontusion zurückzuführen, befriedigt nun weder das klinische noch das anatomische Bedürfnis. Wissen wir aus tausendfacher Erfahrung, daß ein peripherer Nerv durch einfache Kontusion oder gar Fernwirkung, also ohne anatomisch nachweisbar beschädigt zu sein, in seiner Funktion wie nach einer Querschnittstrennung betroffen sein kann und daB diese.Erscheinungen vom A ugenblick der Verletzung an bestehen, so dürfen wir um so mehr bei frühen Kontusionsherden im zentralen Nervensystem erwarten, daß die Funktionsstörung in unmittelbarem Anschluß an die Verletzung zum Ausdruck gelange: in unserem Falle aber bestand ein freies Intervall über fast 2 Tage! Die Funktionsstörung trat erst auf im AnschluB an die Operation und war nicht solche einer Hirnstörung, etwa nach Carotisunterbindung.

Wenn auch zuzugeben ist, daß obige Krankengeschichte, als $\mathrm{K}$ ri egskrankenges chich te an bewegter Front entstanden, manche Lücke aufweist, so geht doch daraus hervor, daß die Störungen, die zum Tode führten, auf die Halsmarkerweichung zurückzuführen sind. Als komplizierend mag die Blutungsanämie und die rechtsseitige Blutsperre zum Großhirn betrachtet, doch muß in Rechnung gesetzt werden, daß die Carotis interna ja schon durch den Granatsplitter quer durchtrennt war und Blut dem Gehirn kaum mehr zugeführt haben dürfte, also daß der Kollateralkreislauf wohl genügend gearbeitet haben muß. Mir selber kam der Tod ganz überraschend, zumal der Verletzte das Bewußtsein nach der Operation wiedererlangt hatte. Leider fehlt, 
da der Tod innerhalb der ersten 24 Stunden nach der Operation am frühen Morgen eintrat, die $\mathrm{klin}$ is $\mathrm{ch}$ e Beobachtung, ob eine Lähmung peripher des 4. Halsmarkquerschnittes am Rumpf und an den Extremitäten bestand. Aber das ist wohl bei der Art des anatomischen Befundes der Querdurchschnittserweichung als sicher anzunehmen. Also bestand a $\mathrm{ch} \mathrm{Ph}$ renikusläh mung und darf die Atemnot bzw. das schwere Atmen mit Lungenödem und Tod sich daraus erklären.

Die Ursachef ürden Erweichungsherdam Hals. mark suche ich in der Schädigung der A. vertebralis, die für die Blutversorgung des Halsmarks wichtig ist. Dieser Gedanke kam mir in Erinnerung an einen „Fall von is chä mi scher R ückenmarksaffektion bei tuberkulöser Spondylitis (s. Wiet ing, Deutsche Zeitschr. f. Chir., Bd. 70), in dem bei starkem Gibbus ein fast obturierender Thrombus in der abgeknickten Aorta mit anschließender Thrombusbildung in beiden Io. Interkostalarterien eine abwärtige Lähmung herbeigeführt hatte.

Die A. A. vertebrales geben die beiden A. A. spinales posteriores und die zu einer Arteria sulci (anterior) sich vereinigenden A. A. spin. ant. ab, die bis zum Filum terminale hinabziehen. Die A. A. vertebrales bilden aber auch den Ursprung für die durch die Zwischenwirbellöcher eintretenden segmentalen $R$ a mi spi. nales des Halsmarkes bis zum 4. Segment hinunter, bildenalsoindiesem Bezirkdie einzige Blutquelle $f \ddot{u} r d i e s e s$. (Siehe unter anderen $T o l d t$, Anatomischer Atlas: Die Gefäßlehre, Fig. 980 u. 981 ).

Es ist nun sehr wohl möglich, daß, sei es spontan oder durch die Tamponade bei der Operation, die dadurch verschlossene rechtsseitige Arteria vertebralis thrombosierte und daB durch Wachstum des sekundären Throm. bus die Blutzufuhr am Halsmark aufgehoben w u rd e. Die Kollateralen von der anderen Seite her, zumal wenn die einzige Art. sulci, aus der die Vasocorona vornehmlich gespeist wird, thrombotisch verschlossen war, genügten nicht mehr zur Aufrechterhaltung der Ernährung.

Für das Rückenmark ist die Möglichkeit dieser Genese von Erweichungshorden kaum beachtet worden und kann ich 
Schmaus' Bemerkung (s. seine Monographie: „Die Kompressionsmyelitis bei Caries der Wirbelsäule." Wiesbaden 1889), die sich allerdings auf Rückgratstuberkulosen bezieht, nur unterstreichen: „Zur ihr (der Kompression), ebenfalls eine mechanische Ursache darstellend, gehört noch eine Tatsache, von der es nur zu verwundern ist, daß sie in der Literatur so wenig in dieser Beziehung verwortet wurde: $\mathrm{Da}$ die Äste der Arteria sulci und der Vasocorona Endarterien sind, so muß jede Thrombose und Embolieindenselben zuranämischen Erweichung eines bestimmten Gewebsbezirkes führen."

Wenn auch die Autopsie meines Falles - die Gegend ist ja anatomisch schwer zugänglich, makroskopische und mikroskopische Untersuchung fehlt, da an diese Genese nicht gedacht wurde, sondern wir unsi mit der „Fernkontusion“ behalfen - keine Unterlage für die Gefäßveränderungen bietet, möchte ich doch ihr Bestehen als die Markerweichung auslösend, annehmen, und glaube, daß wohl a u ch manche andere „Kontusionsherdedes Rückenmarks" auf solcher Gefäßschädigung beruhen möchten.

Die Bedingungen der auf- und absteigenden Thrombose sind ja zur Genüge gegeben: I $\mathrm{n}$ e $\mathrm{kt}$ i on als Grundlage für die Thrombenbildung im zerrissenen Gefäß, Empfindlichkeit des Endorgans, allgemeine Anämie. An eine Embolie glaube ich nicht, da dievis a tergofehlt.

In der inneren Medizin (siehe: Handbuch der praktischen Medizin, Ebste in-S chwalbe, unter $\mathrm{Z}$ iehen: Krankheiten des Nervensystems) sind embolische und thrombotische Vorgänge im verlängerten Mark beschrieben, in denen wir Analoga finden könnten (siehe S. 85 Akute embolische Bulbärparalyse). Sonst ist unter Apoplexie spin. fast stets eine Blutung verstanden.

Auffallenderweise ist in der C:hirurgieder Verletzungen der $\mathrm{Halsg}$ ef ä $\mathrm{Be}$ anscheinend an solche Folgezustände einer Vertebralisversehrung kaum gedacht worden. Ich habe unter anderem $H$. $K \ddot{u} t \mathrm{t} n$ ers eingehend sonst alle Punkte der Vertebralisverletzung behandelnde Arbeit (s. Bruns' Beiträge, Bd. Io8, H. I : ,Die Verletzungen und traumatischen Aneurysmen der Vertebralgefäße am Halse und ihre operative Behandlung 1917) auf diese Dinge hin genauer durchgesehen und glaube unter den dort 
verzeichneten 26 Fällen $^{1}$ ) eigentlich nur den von A. Bier operierten zu meinem Thema in Beziehung bringen zu können:

Es handelte sich um einen etwa 5 Monate zurückliegenden gut verheilten Gewehrdurchschuß des Halses von $r$. nach 1 . mit kleinem durch die Nekropsie festgestellten Zwerchsackhämatom der lochartig durchschossenen $r$. A. carotis interna mit anschließendem die ganze Carotis interna ausfüllenden älteren $\mathrm{Thrombus}$, und um ein mächtiges der 1 . Halswirbelsäule entlang laufendes $\mathrm{kommunizie.}$ rendes $\mathrm{Hämatom}$ der A. vertebralis sin, das operativ angegangen wurde. Während der O peration trat plötzlich Atemstillstand ein, Tracheotomie und künstliche Atmung hielt das Leben noch bei gutem Pulse etwa 12 Stunden aufrecht, bis auch das Herz versagte. Uber die Versorgung der Gefäßwunde, die nach nekroptischem Befund auf $2 \mathrm{~mm}$ mit dem Hämatom zwischen III. und IV. Querfortsatz kommunizierte, ist nichts gesagt, ebenso nicht, ob das Bewußtsein zurïckkehrte. Betreffs der Atmung "lag genau der Zustand vor wie man ihn bei der Atemstörung nach Operation von Kleinhirnbrückengeschwülsten findet." Es bestanden vor dem Tode Pupillendifferenzen ohne Lichtreaktion, Ausfall aller Reflexe vom Bauche abwärts, soweit sie geprüft wurden. - Die A u topsie ergab außer den angedeuteten Befunden eine $f$ ris $c h$ e $T h$ rombose vom SchuBloch derl. Vertebralis a uf ärts bis indie r. Vertebralis und über den Circulus Willisi hinaus in die rechte Carotis interna, sich hier dem älteren Throm. bus anschließend. - Uber einen Befund am Gehirn wie am Halsmark ist nichts vermerkt. Todesursache: Atemlähmung.

Wenn auch $\mathrm{K} u ̈ t \mathrm{tne} r$ darin recht zu geben ist, daß der Kollateralkreislauf der Vertebralarterien anatomisch vorzüglich ist, vielleicht mit der Einschränkung: wenigstens in ihrem zum Gehirn führenden Teile, lehrt der Fall Biers, daß doch einmal ausgedehnte Thrombosen dort vorkommen können. Leider fehlen auch in diesem Falle wie in allen mir vorliegenden Berichten Angaben über die Gefäßverhältnisse am Halsmark, ob nicht auch sie, also zunächst ihre segmentalen spinalen Äste, thrombosiert waren. Es wäre aber wohl wünschenswert, daß bei allen sog. Kontusionsherden des Rückenmarksden Gefä Ben

1) Ich darf auf zwei weitere dort nicht berücksichtigte Fälle von kom. munizierendem Hämatom der Vertebralis aufmerksam machen, die ich in: Vollbrecht.Wieting, ,Kriegsärztliche Erfahrungen aus dem Balkankriege I914," Fischer-Kornfeld, Berlin, kurz beschrieb, und auf einen dritten von Verte. bralisverletzung bei Resektion des Atlasbogens wegen tuberkulöser Spondylarthritis, den ich am Schlusse kurz berühre. 
diegebührende A uf merksamkeitgeschenktwürde und im $Z$ weifelsfalle a uch mikroskopische Untersuchung der Randgefäße auf Wand und Inhalt stattfände. Die nach der Operation beobachteten Erscheinungen von seiten des Zentralnervensystems sind im Bierschen Falle zu wenig eindeutig, um nachträglich noch auf eine bestimmte anatomische Grundlage zurückgeführt werden zu können. Was sich sonst in der Literatur finden lieb, war nicht zu verwerten.

Therapeutisch werden sich besondere Maßnahmen aus dem Mitgeteilten nicht herleiten lassen. Daß die Vermeid ung jeg licher Infektion mit sekundärer Thrombenbildung ganz wesentlich ist für die primäre Thrombenbildung, ist genugsam bekannt oder sollte es wenigstens sein. Ich spreche wenigstens der Infektion eine weit größere Bedeutung $z$ u als der Art des GefäBverschlusses, auf die Perthes (s. Archiv f. klin. Chir. 1920, Bd. I 44 und Vortrag auf dem Chirurgenkongre $B$ I 920) neuerdings für den Carotisverschlu $B$ wieder so großen Wert legt. Eine $G$ ef $\ddot{a} B n$ aht wird für den größten Teil des Verlaufes der A. vertebralis unmöglich und, wie $K \ddot{u} t t$. ner betont, auch unnötig sein.

Zwecks Stillung der primären und sekundären Blutung aus der Vertebralis ist auffallend selten von der Klemmethode mit Liegenlassen der Klemme Gebrauch gemacht. Ich habe dies Verfahren in einem Falle von Vertebralisblutung bei Atlasresektion, also aus Strecke III $K$ ü $t$ ners, mit Erfolg und ohne böse Folgen angewandt, und verwende sie seit vielen Jahren gleich Garrè auch sonst in geeig. neten Fällen nicht selten, wo Unterbindungen oder Umstechungen schwierig oder nicht sicher genug sind, und dann entweder allein, oder zur Sicherung mir nicht ganz sicherer Unterbindungen. Im Auslande (Frankreich) scheint dies Verfahren gebräuchlicher zu sein. 